\title{
APPENDIX II. CARBON/CARBONATE AND NITROGEN ANALYSES, LEG 64, GULF OF CALIFORNIA ${ }^{1}$
}

Bernd R. T. Simoneit, ${ }^{2}$ Institute of Geophysics and Planetary Physics, University of California, Los Angeles, California and

G. W. Bode, Scripps Institution of Oceanography, La Jolla, California

We analyzed Leg 64 sediments for total carbon and acid-insoluble (organic) carbon using a LECO WR-12 Analyzer. We first dried the $3-5 \mathrm{~cm}^{3}$ sediment samples at $105^{\circ}-110^{\circ} \mathrm{C}$ (some shallow sediments used for the C-H-N analyses were dried at $45-55^{\circ} \mathrm{C}$ ) and then ground them to a homogeneous powder. The ground sediment was redried and two samples $(0.1 \mathrm{~g}$ and $0.5 \mathrm{~g})$ were then weighed in LECO clay crucibles. The $0.5-\mathrm{g}$ sample was acidified with a $10 \%$ hydrochloric acid solution and then washed with distilled water. We then dried and analyzed the sample for acid-insoluble carbon, listed in Table 1 as "organic" carbon. The 0.1-g sample was analyzed for total carbon without further treatment. If the result showed less than $10 \% \mathrm{CaCO}_{3}$, we analyzed an additional 0.5 -g sample for greater accuracy.

We calculated the calcium carbonate percentages as follows: ( $\%$ total $\mathrm{C}-\%$ organic $\mathrm{C}) \times 8.33=\%$ $\mathrm{CaCO}_{3}$. Although other carbonates may be present, all acid-soluble carbon was calculated as calcium carbonate. All results are given in weight percentage (Table 1).

The calcium carbonate values listed in parentheses in Table 1 were determined on shipboard by the carbonate bomb method. Detailed descriptions of these techniques and theory may be found in Bader et al. (1970) and Boyce and Bode (1972).

For all samples analyzed onboard by the C-H-N method, the nitrogen content was also calculated from the data (Table 1). We computed the atomic $\mathrm{C} / \mathrm{N}$ ratio using the organic carbon and nitrogen values (Table 1). This ratio was useful in assessing the thermal maturity of the sedimentary organic matter.

At various intervals during the cruise and on shore, we analyzed standard sediments for calibration. The precision of the method for carbon and nitrogen was the same as we reported for carbon (Boyce and Bode, 1972).

\section{ACKNOWLEDGMENT}

We thank J. Pine and the staff of the Deep Sea Drilling Project core analysis laboratory for these data. Institute of Geophysics and Planetary Physics, UCLA, Contribution 2097.

\section{REFERENCES}

Bader, R. G., Gerard, R. D., et al., 1970. Init. Repts. DSDP, 4: Washington (U.S. Govt. Printing Office).

Boyce, R. E., and Bode, G. W., 1972. Carbon and carbonate analyses, Leg 9, Deep Sea Drilling Project. In Hays, J. D., et al., Init. Repts. DSDP, 9: Washington (U.S. Govt. Printing Office), 797-816.

\footnotetext{
${ }^{1}$ Curray, J. R., Moore, D. G., et al., Init, Repts. DSDP, 64: Washington (U.S. Govt. Printing Office).

2 Present address: School of Oceanography, Oregon State University, Corvallis, Oregon.
}

Table 1. Carbon and nitrogen analyses of samples from Leg 64.

\begin{tabular}{|c|c|c|c|c|c|c|}
\hline $\begin{array}{c}\text { Sample } \\
\text { (interval in } \mathrm{cm} \text { ) }\end{array}$ & $\begin{array}{l}\text { Sub-bottom } \\
\text { Depth } \\
\text { (m) }\end{array}$ & $\begin{array}{c}\text { Total } \\
\text { Carbon } \\
(\%)\end{array}$ & $\begin{array}{c}\text { Organic } \\
\text { Carbon } \\
(\%)\end{array}$ & $\begin{array}{c}\mathrm{CaCO}_{3}{ }^{\mathrm{a}} \\
(\%)\end{array}$ & $\begin{array}{c}\text { Organic } \\
\text { Nitrogen } \\
(\%)\end{array}$ & $\begin{array}{c}\text { Atomic } \\
\mathrm{C} / \mathrm{N}\end{array}$ \\
\hline $474-1-2,39$ & 1.89 & 3.4 & 2.6 & 6 & & \\
\hline $2-2,5$ & 3.55 & 3.5 & 2.2 & 11 & & \\
\hline $3-2,48$ & 13.48 & 5.0 & 2.5 & 21 & & \\
\hline $4-2,79$ & 23.29 & 5.9 & 3.1 & 23 & & \\
\hline $5-1,55$ & 31.05 & & & (7) & & \\
\hline $5-3,46$ & 33.96 & 4.2 & 2.6 & 13 & & \\
\hline $5-3,105$ & 44.05 & 4.7 & 2.8 & (16) & 0.22 & 15 \\
\hline $6-4,41$ & 44.91 & & & (17) & & \\
\hline $6-4,68$ & 45.18 & 3.9 & 1.1 & 24 & & \\
\hline $6-5,32$ & 46.32 & 1.5 & 1.1 & (3) & 0.11 & 12 \\
\hline $6-6,50$ & 48.00 & & & (38) & & \\
\hline $7-3,51$ & 53.01 & & & (20) & & \\
\hline $7-5,109$ & 56.59 & 3.2 & 0.3 & 25 & & \\
\hline $8-2,49$ & 60.99 & 2.6 & 0.6 & 17 & & \\
\hline $8-5,38$ & 65.38 & & & (9) & & \\
\hline $11-2,12$ & 89.12 & 3.6 & 2.8 & 6 & & \\
\hline $12-1,38$ & 97.38 & 4.4 & 2.5 & 15 & & \\
\hline $13-1,47$ & 106.97 & 3.0 & 2.0 & 8 & & \\
\hline $13-1,104$ & 107.54 & & & (17) & & \\
\hline $14-2,45$ & 117.95 & 2.7 & 2.5 & 2 & & \\
\hline $14-2,56$ & 118.06 & & & (1) & & \\
\hline $16-3,0$ & 138.00 & 5.0 & 2.8 & 18 & & \\
\hline $16-3,8$ & 138.08 & 1.4 & 0.8 & 5 & & \\
\hline $17-1,7$ & 144.57 & 3.7 & 1.4 & 19 & & \\
\hline $17-1,75$ & 145.25 & & & (22) & & \\
\hline $17-5,49$ & 150.99 & & & (21) & & \\
\hline $17-6,93$ & 152.93 & 2.5 & 1.6 & (7) & 0.15 & 12 \\
\hline $18-3,36$ & 157.36 & 4.0 & 1.4 & 22 & & \\
\hline $19-2,89$ & 165.89 & 3.3 & 2.1 & 10 & & \\
\hline $474 \mathrm{~A}-1-4,7$ & 168.07 & 2.4 & 1.7 & 6 & & \\
\hline $1-4,78$ & 168.78 & & & (4) & & \\
\hline $1-4,84$ & 168.84 & 2.0 & 1.6 & 3 & & \\
\hline $1-4,99$ & 168.99 & 1.7 & 0.1 & $13(9)$ & & \\
\hline $2-1,111$ & 174.11 & 1.3 & 0.6 & $6(9)$ & & \\
\hline $2-1,135$ & 174.35 & 0.8 & 0.4 & 4 & & \\
\hline $3-5,33$ & 188.83 & 1.8 & 1.5 & 3 & & \\
\hline $3-5,76$ & 189.26 & 3.2 & 1.5 & 14 & & \\
\hline $3-5,109$ & 189.59 & 3.4 & 0.6 & 24 & & \\
\hline $3-5,114$ & 189.64 & & & (24) & & \\
\hline $3-5,132$ & 189.82 & 4.0 & 0.6 & 28 & & \\
\hline $4-4,71$ & 197.21 & & & (5) & & \\
\hline $4-4,75$ & 197.25 & 1.8 & 1.4 & 3 & & \\
\hline $6-1,97$ & 211.97 & 3.4 & 1.6 & 15 & & \\
\hline $6-1,147$ & 212.47 & 2.5 & 0.5 & 17 & & \\
\hline $6-1,148$ & 212.48 & & & (20) & & \\
\hline $7-2,107$ & 223.07 & 2.2 & 1.4 & (6) & 0.12 & 15 \\
\hline $7, \mathrm{CC}$ & 224.68 & 2.2 & 1.8 & 3 & & \\
\hline $8-5,67$ & 236.67 & & & (20) & & \\
\hline $9-3,61$ & 243.11 & 3.8 & 2.2 & 13 & & \\
\hline $9-4,55$ & 244.55 & & & (12) & & \\
\hline $10-2,62$ & 251.12 & 2.1 & 1.7 & 3 & & \\
\hline $10-4,58$ & 255.08 & & & (22) & & \\
\hline $12-3,6$ & 271.06 & 3.4 & 1.9 & 12 & & \\
\hline $12-4,121$ & 273.71 & 2.4 & 1.7 & (6) & 0.14 & 14 \\
\hline $13-3,58$ & 281.08 & & & (19) & & \\
\hline $13-4,115$ & 283.15 & 4.0 & 1.9 & 18 & & \\
\hline $16-3,65$ & 309.65 & 2.8 & 1.8 & 8 & & \\
\hline $17-4,81$ & 320.81 & 2.7 & 1.8 & 7 & & \\
\hline $19-2,48$ & 328.98 & 1.7 & 1.4 & 2 & & \\
\hline $19-2,55$ & 329.05 & & & (5) & & \\
\hline $21-5,76$ & 350.76 & & & (7) & & \\
\hline $21-5,77$ & 350.77 & 1.1 & 0.7 & 3 & & \\
\hline $21-6,46$ & 351.96 & 2.3 & 1.7 & (5) & 0.14 & 14 \\
\hline $23-1,92$ & 363.92 & 2.1 & 1.3 & 6 & & \\
\hline $23-4,85$ & 368.35 & 2.3 & 2.0 & 2 & & \\
\hline $23-4,112$ & 368.62 & 2.4 & 1.6 & 7 & & \\
\hline $24-2,137$ & 375.37 & 2.5 & 1.5 & (8) & 0.11 & 15 \\
\hline
\end{tabular}


Table 1. (Continued).

\begin{tabular}{|c|c|c|c|c|c|c|}
\hline $\begin{array}{c}\text { Sample } \\
\text { (interval in } \mathrm{cm} \text { ) }\end{array}$ & $\begin{array}{l}\text { Sub-bottom } \\
\text { Depth } \\
\text { (m) }\end{array}$ & $\begin{array}{c}\text { Total } \\
\text { Carbon } \\
(\%)\end{array}$ & $\begin{array}{c}\text { Organic } \\
\text { Carbon } \\
(\%)\end{array}$ & $\underset{(\%)}{\mathrm{CaCO}_{3}}$ & $\begin{array}{c}\text { Organic } \\
\text { Nitrogen } \\
(\%)\end{array}$ & $\begin{array}{l}\text { Atomic } \\
\mathrm{C} / \mathrm{N}\end{array}$ \\
\hline $474 A-25-1,58$ & 382.58 & 3.2 & 2.7 & 5 & & \\
\hline $25-1,140$ & 383.40 & 3.5 & 2.4 & (9) & 0.18 & 15 \\
\hline $25-2,15$ & 383.65 & & & (10) & & \\
\hline $26-1,100$ & 392.50 & 4.6 & 2.6 & 16 & & \\
\hline $27-3,78$ & 404.78 & & & (28) & & \\
\hline $27-4,79$ & 406.29 & 2.2 & 1.7 & 5 & & \\
\hline $28-2,119$ & 413.19 & 2.9 & 1.7 & (9) & 0.15 & 13 \\
\hline $28-4,74$ & 415.74 & 2.3 & 1.3 & 8 & & \\
\hline $29-1,44$ & 420.44 & & & (9) & & \\
\hline $29-2,110$ & 422.60 & 3.5 & 2.3 & 10 & & \\
\hline $30-1,69$ & 430.19 & & & (9) & & \\
\hline $30-3,67$ & 433.17 & 3.3 & 1.6 & 14 & & \\
\hline $31-3,53$ & 442.53 & 2.5 & 1.8 & 6 & & \\
\hline $32-2,120$ & 451.20 & 3.0 & 1.9 & (9) & 0.14 & 16 \\
\hline $32-5,107$ & 455.57 & 2.5 & 2.0 & 4 & & \\
\hline $32-7,20$ & 457.70 & & & (6) & & \\
\hline $33-3,81$ & 461.81 & 2.1 & 1.4 & 6 & & \\
\hline $35-1,22$ & 477.22 & 4.0 & 2.4 & (13) & 0.18 & 16 \\
\hline $36-2,107$ & 489.07 & 4.4 & 2.3 & 17 & & \\
\hline $37-3,100$ & 500.00 & 3.4 & 2.2 & (10) & 0.16 & 16 \\
\hline $39-1,59$ & 515.59 & 0.7 & 0.5 & 2 & & \\
\hline $39-3,29$ & 518.29 & 1.6 & 1.1 & (4) & 0.09 & 13 \\
\hline $39-3,51$ & 518.51 & & & (8) & & \\
\hline $39-3,111$ & 519.11 & 1.7 & 0.9 & (6) & 0.08 & 12 \\
\hline $40-2,136$ & 527.36 & 2.2 & 1.6 & (5) & 0.11 & 16 \\
\hline $40-3,5$ & 527.55 & 1.1 & 0.6 & (4) & 0.06 & 12 \\
\hline $40-3,19$ & 527.69 & & & (38) & & \\
\hline $41-1,120$ & 535.20 & 1.6 & 0.4 & (10) & 0.04 & 12 \\
\hline $41-3,119$ & 538.19 & 1.5 & 0.7 & (6) & 0.08 & 12 \\
\hline $475-1-2,61$ & 2.11 & & & (9) & & \\
\hline $1-2,67$ & 2.17 & 2.6 & 1.9 & 6 & & \\
\hline $2-2,127$ & 8.77 & 3.0 & 2.6 & (3) & 0.26 & 12 \\
\hline $2-3,65$ & 9.65 & 4.6 & 2.8 & 15 & & \\
\hline $2-4,6$ & 10.56 & 3.7 & 2.8 & 8 & & \\
\hline $2-4,16$ & 10.66 & & & (9) & & \\
\hline $2-5,12$ & 12.12 & 3.3 & 1.7 & 13 & & \\
\hline $3-2,70$ & 17.70 & 4.1 & 1.7 & (19) & 0.16 & 12 \\
\hline $3-2,71$ & 17.71 & 3.3 & 1.3 & 16 & & \\
\hline $5-3,70$ & 38.20 & & & (11) & & \\
\hline $5-4,7$ & 39.07 & 1.7 & 1.4 & 2 & & \\
\hline $6-2,50$ & 46.00 & 2.1 & 1.7 & (3) & 0.15 & 13 \\
\hline $6-2,53$ & 46.03 & & & (5) & & \\
\hline $6-5,24$ & 50.24 & 3.2 & 2.1 & 9 & & \\
\hline $6-5,115$ & 51.15 & 1.9 & 1.5 & (3) & 0.12 & 14 \\
\hline $8-5,62$ & 69.62 & 2.1 & 2.1 & 0 & & \\
\hline $8-5,133$ & 70.33 & 1.3 & 1.2 & (1) & 0.10 & 14 \\
\hline $8-6,22$ & 70.72 & 3.7 & 3.3 & (3) & 0.25 & 15 \\
\hline $9-3,29$ & 75.79 & & & (5) & & \\
\hline $9-4,7$ & 77.07 & 2.5 & 2.5 & 0 & & \\
\hline $11-4,29$ & 96.29 & & & (1) & & \\
\hline $11-6,15$ & 99.15 & 1.9 & 1.7 & 2 & & \\
\hline $12-4,117$ & 106.67 & 1.4 & 1.1 & (2) & 0.10 & 13 \\
\hline $12-5,29$ & 107.29 & 2.3 & 1.3 & 8 & & \\
\hline $13-4,48$ & 115.48 & & & (5) & & \\
\hline $13-4,49$ & 115.49 & 2.2 & 1.8 & 4 & & \\
\hline $15-2,79$ & 131.79 & 1.4 & 1.3 & 1 & & \\
\hline $15-2,122$ & 132.22 & & & (3) & & \\
\hline $15-4,53$ & 134.53 & 2.8 & 2.4 & (3) & 0.16 & 17 \\
\hline $15-6,68$ & 137.68 & 2.3 & 2.3 & 0 & & \\
\hline $16-2,104$ & 141.54 & 2.4 & 2.1 & (2) & 0.15 & 16 \\
\hline $16-5,35$ & 145.35 & 1.5 & 1.2 & 3 & & \\
\hline $17-1,70$ & 149.20 & 2.3 & 2.1 & (2) & 0.15 & 16 \\
\hline $17-2,46$ & 150.46 & 1.0 & 1.0 & 0 & & \\
\hline $17-3,29$ & 151.79 & 0.9 & 0.9 & 0 & & \\
\hline $17-4,45$ & 153.45 & 2.2 & 1.9 & 3 & & \\
\hline $17-4,131$ & 154.31 & 1.8 & 1.8 & (0) & 0.14 & 15 \\
\hline $17-5,5$ & 154.55 & 2.5 & 2.4 & (1) & 0.18 & 16 \\
\hline $475 B-1-5,39$ & 6.39 & 3.6 & 2.8 & (7) & 0.23 & 14 \\
\hline $1-5,42$ & 6.42 & & & (6) & & \\
\hline $1-7 ; 59$ & 9.59 & 3.4 & 1.4 & 16 & & \\
\hline $476-1-2,61$ & 2.11 & & & (5) & & \\
\hline $1-2,147$ & 2.97 & 3.6 & 2.3 & 10 & & \\
\hline $2-2,19$ & 10.69 & 2.1 & 1.5 & (6) & 0.14 & 12 \\
\hline $2-3,75$ & 12.75 & & & (13) & & \\
\hline $2-4,135$ & 14.85 & 4.6 & 3.1 & 13 & & \\
\hline $3-3,88$ & 22.38 & 4.4 & 3.2 & 10 & & \\
\hline $3-4,120$ & 24.20 & 3.2 & 2.7 & (4) & 0.22 & 14 \\
\hline $3-6,51$ & 26.51 & & & (21) & & \\
\hline $4-3,5$ & 31.05 & 2.9 & 2.5 & 3 & & \\
\hline $4-5,26$ & 34.26 & & & (12) & & \\
\hline $5-3,75$ & 41.25 & 4.6 & 0.7 & 32 & & \\
\hline $5-3,136$ & 41.86 & 2.9 & 1.3 & (12) & 0.12 & 12 \\
\hline $5-3,138$ & 41.88 & & & (12) & & \\
\hline
\end{tabular}

Table 1. (Continued).

\begin{tabular}{|c|c|c|c|c|c|c|}
\hline $\begin{array}{c}\text { Sample } \\
\text { (interval in cm) }\end{array}$ & $\begin{array}{l}\text { Sub-bottom } \\
\text { Depth } \\
\text { (m) }\end{array}$ & $\begin{array}{c}\text { Total } \\
\text { Carbon } \\
(\%)\end{array}$ & $\begin{array}{c}\text { Organic } \\
\text { Carbon } \\
(\%)\end{array}$ & $\underset{(\%)}{\mathrm{CaCO}_{3}}$ & $\begin{array}{c}\text { Organic } \\
\text { Nitrogen } \\
(\%)\end{array}$ & $\begin{array}{l}\text { Atomic } \\
\mathrm{C} / \mathrm{N}\end{array}$ \\
\hline $476-6-2,32$ & 48.82 & & & (13) & & \\
\hline $6-4,69$ & 52.19 & 5.8 & 2.6 & 26 & & \\
\hline $7-2,103$ & 59.03 & & & (40) & & \\
\hline $7-4,12$ & 61.12 & 1.5 & 1.4 & 1 & & \\
\hline $7-6,108$ & 65.08 & 2.6 & 1.8 & (7) & 0.15 & 14 \\
\hline $8-2,124$ & 68.74 & 2.8 & 1.6 & 10 & & \\
\hline $9-2,52$ & 77.52 & 2.0 & 1.5 & (4) & 0.13 & 14 \\
\hline $10-4,6$ & 89.56 & 2.3 & 2.3 & 0 & & \\
\hline $10-6,114$ & 93.64 & 3.5 & 3.2 & (2) & 0.22 & 17 \\
\hline $11-4,109$ & 100.09 & 2.1 & 1.8 & (3) & 0.14 & is \\
\hline $11-6,98$ & 102.98 & 2.4 & 2.1 & 2 & & \\
\hline $13-1,61$ & 114.11 & 1.2 & 0.9 & (2) & 0.08 & 13 \\
\hline $15-2,108$ & 135.08 & 1.1 & 0.9 & (1) & 0.09 & 12 \\
\hline $15-3,75$ & 136.25 & 1.3 & 1.2 & 1 & & \\
\hline $16-3,69$ & 145.69 & 1.2 & 1.1 & $i$ & & \\
\hline $17-1,87$ & 152.37 & 2.3 & 1.9 & 3 & & \\
\hline $17-3,82$ & 155.32 & 2.2 & 1.7 & (4) & 0.14 & 14 \\
\hline $18-5,40$ & 167.40 & & & (4) & & \\
\hline $18-5,67$ & 167.67 & 2.0 & 1.7 & 2 & & \\
\hline $18-6,88$ & 169.38 & 2.2 & 1.4 & (3) & 0.10 & 17 \\
\hline $19-1,88$ & 171.38 & 1.6 & 1.6 & 0 & & \\
\hline $19-1,96$ & 171.46 & & & (1) & & \\
\hline $19-1,106$ & 171.56 & 1.8 & 1.4 & (3) & 0.10 & 16 \\
\hline $20-3,120$ & 184.20 & & & (11) & & \\
\hline $20-4,48$ & 184.98 & 3.2 & 1.6 & (12) & 0.13 & 14 \\
\hline $20-5,71$ & 186.71 & 3.2 & 1.6 & 13 & & \\
\hline $21-3,3$ & 192.53 & 1.1 & 1.0 & 1 & & \\
\hline $21-3,46$ & 192.96 & 0.4 & 0.4 & (0) & 0.03 & 17 \\
\hline $21-4,43$ & 194.43 & 2.9 & 2.9 & (0) & 0.22 & 15 \\
\hline $21, \mathrm{CC}$ & 194.55 & 7.1 & 7.1 & (0) & 0.31 & 27 \\
\hline $477-2-2,136$ & 3.86 & 2.8 & 2.1 & (6) & 0.22 & 11 \\
\hline $3-2,1$ & 12.01 & 2.8 & 2.0 & 7 & & \\
\hline $3-2,28$ & 12.28 & 4.2 & 3.3 & (8) & 0.35 & 11 \\
\hline $3-2,83$ & 12.83 & & & (11) & & \\
\hline $3-2,115$ & 13.15 & 3.8 & 2.6 & 10 & & \\
\hline $4-1,30$ & 20.30 & 4.2 & 3.2 & (8) & 0.32 & 12 \\
\hline $4-1,43$ & 20.43 & & & (8) & & \\
\hline $4-1,84$ & 20.84 & 3.7 & 2.5 & 10 & & \\
\hline $5-1,80$ & 30.30 & 3.6 & 2.1 & 11 & & \\
\hline $5-1,89$ & 30.39 & 3.6 & 2.6 & (8) & 0.24 & 13 \\
\hline $5-1,94$ & 30.44 & & & (9) & & \\
\hline $5, \mathrm{CC}$ & 39.00 & 3.9 & 2.6 & (10) & 0.19 & 16 \\
\hline $7-1,27$ & 48.77 & 3.3 & 2.4 & 7 & & \\
\hline $7-1,124$ & 49.74 & 2.8 & 1.8 & (8) & 0.10 & 21 \\
\hline $7-2,14$ & 50.14 & 1.3 & 0.7 & (4) & 0.03 & 27 \\
\hline $15-1,28$ & 105.28 & 2.2 & 1.1 & (1) & 0.07 & 17 \\
\hline $16-1,36$ & 115.36 & 1.1 & 0.8 & (3) & 0.07 & 14 \\
\hline $16-2,29$ & 116.79 & 1.2 & 1.1 & 1 & & \\
\hline $16-2,71$ & 117.21 & & & (0) & & \\
\hline $16-3,99$ & 118.99 & 1.0 & 0.7 & (0) & 0.07 & 12 \\
\hline $17-1,41$ & 124.91 & 1.0 & 0.7 & (3) & 0.06 & 14 \\
\hline $17-3,29$ & 127.79 & 0.9 & 0.9 & 0 & & \\
\hline $17-3,44$ & 127.94 & 1.0 & 0.8 & (0) & 0.07 & 12 \\
\hline $19-2,138$ & 146.38 & 1.0 & 0.5 & 4 & & \\
\hline $19-3,29$ & 146.79 & 1.1 & 0.3 & (5) & 0.03 & 11 \\
\hline $20-2,51$ & 155.01 & & & (4) & & \\
\hline $20-2,61$ & 155.11 & 1.6 & 1.0 & (4) & 0.04 & 28 \\
\hline $20-2,88$ & 155.38 & 1.4 & 1.4 & 0 & & \\
\hline $22-1,26$ & 172.26 & 1.6 & 0.8 & (7) & 0.02 & 47 \\
\hline 477 A-5-1, 44 & 191.44 & 1.2 & 0.6 & (6) & 0.01 & 69 \\
\hline $7-1,43$ & 210.43 & 0.3 & 0.2 & (3) & 0.00 & $\infty$ \\
\hline $9-1,39$ & 229.39 & 0.5 & 0.4 & (3) & 0.00005 & 8633 \\
\hline $\begin{array}{r}\text { 477B-1-1, } 14 \\
1-2,110\end{array}$ & $\begin{array}{l}0.14 \\
2.60\end{array}$ & $\begin{array}{l}2.6 \\
2.8\end{array}$ & $\begin{array}{l}1.9 \\
2.1\end{array}$ & $\begin{array}{l}\text { (5) } \\
(6)\end{array}$ & $\begin{array}{l}0.19 \\
0.22\end{array}$ & 12 \\
\hline $1-2,110$ & 2.60 & 2.8 & 2.1 & (6) & 0.22 & 12 \\
\hline $478-1-1,15$ & $\begin{array}{l}0.15 \\
2.47\end{array}$ & 2.3 & 1.7 & $\stackrel{5}{9}$ & & \\
\hline $1-2,97$ & 2.47 & 2.8 & 1.9 & (7) & 0.18 & 12 \\
\hline $2-2,2$ & 5.02 & 4.3 & 3.1 & (10) & 0.30 & 12 \\
\hline $2-3,65$ & 7.15 & 4.6 & 2.8 & 15 & & \\
\hline $2-3,78$ & 7.28 & 2.2 & 1.9 & 3 & & \\
\hline $2-6,102$ & 12.02 & 4.6 & 3.6 & (8) & 0.38 & 11 \\
\hline $3-1,19$ & 13.19 & 2.1 & 1.4 & (6) & 0.11 & 15 \\
\hline $4-6,121$ & 31.21 & 1.5 & 1.3 & (2) & 0.09 & 16 \\
\hline $4, \mathrm{CC}$ & 32.00 & 1.8 & 1.2 & (5) & 0.09 & 15 \\
\hline $5-6,133$ & 40.83 & 1.7 & 1.2 & (4) & 0.08 & 17 \\
\hline $6-3,33$ & 44.83 & 2.4 & 2.2 & (2) & 0.17 & is \\
\hline $8-3,27$ & 63.77 & 2.5 & 1.5 & (8) & 0.12 & 15 \\
\hline $9-1,107$ & 71.07 & 3.4 & 2.1 & (11) & 0.19 & 13 \\
\hline $9-3,107$ & 74.07 & 4.0 & 2.3 & (14) & 0.20 & 13 \\
\hline $10-1,93$ & 80.43 & 2.8 & 2.0 & (7) & 0.17 & 13 \\
\hline $11-4,107$ & 94.57 & 2.2 & 1.5 & (6) & 0.12 & 14 \\
\hline $12-2,81$ & 100.81 & 3.3 & 2.5 & (6) & 0.20 & is \\
\hline $13-1,138$ & 109.38 & 3.0 & 2.4 & (5) & 0.18 & 15 \\
\hline $13-2,116$ & 110.66 & 3.3 & 2.4 & (7) & 0.21 & 13 \\
\hline
\end{tabular}


Table 1. (Continued).

\begin{tabular}{|c|c|c|c|c|c|c|}
\hline $\begin{array}{c}\text { Sample } \\
\text { (interval in } \mathrm{cm} \text { ) }\end{array}$ & $\begin{array}{l}\text { Sub-bottom } \\
\text { Depth } \\
\text { (m) }\end{array}$ & $\begin{array}{c}\text { Total } \\
\text { Carbon } \\
(\%)\end{array}$ & $\begin{array}{c}\text { Organic } \\
\text { Carbon } \\
(\%)\end{array}$ & $\underset{(\%)}{\mathrm{CaCO}_{3}}$ & $\begin{array}{c}\text { Organic } \\
\text { Nitrogen } \\
(\%)\end{array}$ & $\begin{array}{c}\text { Atomic } \\
\mathrm{C} / \mathrm{N}\end{array}$ \\
\hline $478-14-4,118$ & 123.18 & 3.0 & 2.2 & (6) & 0.17 & 15 \\
\hline $15-3,80$ & 130.80 & 2.1 & 2.0 & 1 & & \\
\hline $15-5,41$ & 133.41 & 2.0 & 1.3 & (6) & 0.10 & 16 \\
\hline $16-3,61$ & 140.11 & 1.9 & 1.8 & 1 & & \\
\hline $16-3,67$ & 140.17 & & & 5 & & \\
\hline $16-4,110$ & 142.10 & 1.8 & 1.2 & (5) & 0.09 & 15 \\
\hline $17-3,60$ & 149.60 & 1.6 & 1.2 & 4 & & \\
\hline $17-4,118$ & 151.68 & 1.6 & 0.9 & (6) & 0.06 & 16 \\
\hline $19-6,115$ & 168.15 & 2.6 & 1.8 & (7) & 0.15 & 14 \\
\hline $20-4,102$ & 170.52 & 1.4 & 0.7 & (6) & 0.06 & 13 \\
\hline $21-3,50$ & 178.00 & 3.5 & 2.0 & 12 & & \\
\hline $21-5,140$ & 181.90 & 2.0 & 1.4 & (5) & 0.11 & 15 \\
\hline $22-3,53$ & 187.53 & 2.9 & 1.7 & 10 & & \\
\hline $22-3,97$ & 187.97 & 3.0 & 2.0 & (8) & 0.15 & 15 \\
\hline $28-4,118$ & 248.18 & 2.8 & 1.7 & (9) & 0.16 & 12 \\
\hline $28-6,56$ & 250.56 & 2.8 & 1.3 & (12) & 0.10 & 16 \\
\hline $28-6,90$ & 250.90 & 3.5 & 1.7 & (15) & 0.17 & 12 \\
\hline $29-1,57$ & 251.07 & 4.1 & 2.1 & (16) & 0.16 & 15 \\
\hline $29-1,124$ & 251.77 & 2.8 & 2.2 & (5) & 0.16 & 16 \\
\hline $29-2,34$ & 252.34 & 2.9 & 2.2 & (6) & 0.16 & 16 \\
\hline $29-2,108$ & 253.08 & 2.1 & 1.7 & (3) & 0.12 & 17 \\
\hline $29-2,129$ & 253.29 & 1.4 & 1.4 & (0) & 0.06 & 26 \\
\hline $30-1,44$ & 256.94 & 1.7 & 1.2 & (4) & 0.13 & 11 \\
\hline $30-1,87$ & 257.37 & 3.0 & 2.0 & (8) & 0.14 & 17 \\
\hline $30-2,28$ & 258.28 & 3.1 & 2.3 & (7) & 0.16 & 17 \\
\hline $30-2,140$ & 259.40 & 3.1 & 2.1 & (8) & 0.16 & 15 \\
\hline $31-4,122$ & 265.72 & 2.2 & 2.1 & (9) & 0.17 & 14 \\
\hline $32-2,124$ & 272.24 & 2.7 & 1.9 & (7) & 0.15 & 15 \\
\hline $33-2,55$ & 281.05 & 2.2 & 1.7 & (4) & 0.13 & 15 \\
\hline $34-3,50$ & 292.00 & 3.6 & 2.3 & (ii) & 0.17 & 16 \\
\hline $35-2,77$ & 300.27 & 4.3 & 2.7 & (13) & 0.18 & 17 \\
\hline $36-2,61$ & 309.61 & 3.1 & 1.9 & (10) & 0.15 & 15 \\
\hline $39-3,58$ & 330.08 & 2.6 & 2.3 & (3) & & \\
\hline $39-3,84$ & 330.34 & 2.0 & 1.5 & (4) & 0.09 & 19 \\
\hline $40-2,61$ & 338.11 & 1.1 & 1.1 & 0 & 0.10 & 13 \\
\hline $4791-1,130$ & 1.30 & 4.0 & 3.6 & (3) & 0.39 & 11 \\
\hline $3-2,99$ & 14.99 & 3.2 & 2.8 & (3) & 0.26 & 13 \\
\hline $5-3,107$ & 36.57 & 2.8 & 2.7 & (1) & 0.25 & 13 \\
\hline $6-2,65$ & 43.15 & 4.1 & 2.2 & 16 & & \\
\hline $6-4,80$ & 46.30 & 3.5 & 2.3 & 10 & & \\
\hline $7-5,108$ & 56.58 & 2.7 & 2.7 & (0) & 0.20 & 16 \\
\hline $8-3,45$ & 63.45 & 3.8 & 2.4 & 12 & & \\
\hline $9-3,99$ & 73.49 & 5.3 & 3.0 & (19) & 0.24 & 15 \\
\hline $10-4,65$ & 84.15 & 3.3 & 2.3 & 8 & & \\
\hline $10-6,25$ & 86.75 & 3.1 & 2.8 & 3 & & \\
\hline $10-7,24$ & 89.24 & 2.8 & 2.4 & (3) & 0.21 & 14 \\
\hline $12-6,111$ & 106.61 & 2.9 & 2.4 & (3) & 0.20 & 14 \\
\hline $13-1,110$ & 108.60 & 3.8 & 2.9 & 7 & & \\
\hline $14-2,52$ & 119.02 & 3.2 & 2.6 & (5) & 0.19 & 16 \\
\hline $15-2,97$ & 128.97 & 3.1 & 2.8 & 3 & & \\
\hline $15-5,108$ & 133.58 & 3.2 & 2.9 & 2 & & \\
\hline $16-3,126$ & 140.26 & 3.6 & 3.2 & (3) & 0.24 & 15 \\
\hline $18-1,82$ & 155.82 & 2.0 & 1.9 & 1 & & \\
\hline $21-2,88$ & 185.88 & 2.8 & 2.4 & 3 & & \\
\hline $21-5,110$ & 190.60 & 4.8 & 2.5 & (19) & 0.20 & 15 \\
\hline $22-5,101$ & 200.01 & 2.9 & 2.6 & 3 & & \\
\hline $23-4,73$ & 207.73 & 4.1 & 2.3 & (15) & 0.20 & 13 \\
\hline $24-3,86$ & 215.86 & 2.8 & 2.5 & 2 & & \\
\hline $25-2,131$ & 224.31 & 3.0 & 2.5 & (4) & 0.22 & 13 \\
\hline $26-1,82$ & 231.82 & 2.6 & 1.6 & 9 & & \\
\hline $27-4,72$ & 245.72 & 2.9 & 2.5 & (3) & 0.18 & 16 \\
\hline $29-5,114$ & 266.64 & 3.0 & 2.6 & (3) & 0.20 & 15 \\
\hline $31-5,69$ & 285.19 & 2.6 & 2.6 & (0) & 0.20 & 15 \\
\hline $31-5,70$ & 285.20 & 2.9 & 2.8 & 1 & & \\
\hline $32-1,63$ & 288.63 & 3.5 & 2.5 & 8 & & \\
\hline $34-2,84$ & 309.34 & 3.3 & 2.7 & (5) & 0.22 & 14 \\
\hline $34-5,108$ & 314.08 & 3.3 & 3.0 & 3 & & \\
\hline $35-4,139$ & 322.39 & 3.0 & 2.3 & 5 & & \\
\hline $36-3.70$ & 329.70 & 3.7 & 2.9 & (6) & 0.23 & 15 \\
\hline $37-2,78$ & 337.78 & 2.9 & 2.8 & 1 & & \\
\hline $38-5,129$ & 352.29 & 3.0 & 3.0 & (0) & 0.22 & 16 \\
\hline $39-4,107$ & 360.07 & 3.6 & 3.5 & 1 & & \\
\hline $40-6,110$ & 372.60 & 3.3 & 2.1 & (10) & 0.18 & 14 \\
\hline $41-1,99$ & 374.49 & 3.7 & 2.7 & 8 & & \\
\hline
\end{tabular}

Table 1. (Continued).

\begin{tabular}{|c|c|c|c|c|c|c|}
\hline $\begin{array}{c}\text { Sample } \\
\text { (interval in } \mathrm{cm} \text { ) }\end{array}$ & $\begin{array}{l}\text { Sub-bottom } \\
\text { Depth } \\
\text { (m) }\end{array}$ & $\begin{array}{c}\text { Total } \\
\text { Carbon } \\
\text { (\%) }\end{array}$ & $\begin{array}{c}\text { Organic } \\
\text { Carbon } \\
(\%)\end{array}$ & $\underset{(\%)}{\mathrm{CaCO}_{3}}$ & $\begin{array}{c}\text { Organic } \\
\text { Nitrogen } \\
(\%)\end{array}$ & $\stackrel{\text { Atomic }}{\mathrm{C} / \mathrm{N}}$ \\
\hline $474-43-2,82$ & 394.82 & 3.8 & 2.6 & (10) & 0.22 & 14 \\
\hline $44-3,93$ & 405.93 & 4.1 & 1.9 & 18 & & \\
\hline $44-5,21$ & 408.21 & 3.3 & 1.7 & 13 & & \\
\hline $45-1,81$ & 412.31 & 2.3 & 1.9 & (3) & 0.19 & 11 \\
\hline $47-4,108$ & 436.08 & 2.3 & 1.2 & (9) & 0.17 & 9 \\
\hline $480-3, \mathrm{CC}$ & 14.20 & 3.5 & 2.9 & (5) & 0.27 & 12 \\
\hline 5,CC & 23.60 & 2.8 & 2.8 & (0) & 0.26 & 13 \\
\hline $6, \mathrm{CC}$ & 28.40 & 2.5 & 2.5 & (0) & 0.21 & 14 \\
\hline $7, \mathrm{CC}$ & 33.10 & 3.4 & 2.9 & (4) & 0.24 & 14 \\
\hline $9, \mathrm{CC}$ & 42.60 & 4.2 & 3.0 & (10) & 0.25 & 14 \\
\hline $11, \mathrm{CC}$ & 49.10 & 3.1 & 2.5 & (5) & 0.21 & 14 \\
\hline $13, \mathrm{CC}$ & 66.30 & 2.5 & 2.4 & (1) & 0.20 & 14 \\
\hline $17, \mathrm{CC}$ & 85.40 & 2.7 & 2.7 & (0) & 0.20 & 16 \\
\hline $19, \mathrm{CC}$ & 94.90 & 2.5 & 2.5 & (0) & 0.19 & 15 \\
\hline $21, \mathrm{CC}$ & 104.20 & 4.4 & 3.2 & (10) & 0.23 & 16 \\
\hline $23, \mathrm{CC}$ & 113.90 & 2.2 & 2.2 & (0) & 0.16 & 16 \\
\hline $25, \mathrm{CC}$ & 123.40 & 2.8 & 2.8 & (0) & 0.20 & 16 \\
\hline $27, \mathrm{CC}$ & 132.90 & 2.7 & 2.7 & (0) & 0.20 & 16 \\
\hline $29, \mathrm{CC}$ & 142.40 & 2.4 & 2.3 & (1) & 0.17 & 16 \\
\hline $31, \mathrm{CC}$ & 151.90 & 2.2 & 2.2 & (0) & 0.17 & 15 \\
\hline $481-1-2,57$ & 2.07 & 2.6 & 2.1 & (4) & 0.22 & 11 \\
\hline $2-2,117$ & 7.42 & 4.6 & 1.4 & 26 & & \\
\hline $4-1,85$ & 15.10 & 5.9 & 4.3 & (13) & 0.45 & 11 \\
\hline $7-2,136$ & 31.36 & 3.0 & 2.2 & (6) & 0.22 & 12 \\
\hline $8-2,110$ & 35.85 & 2.4 & 1.7 & 6 & & \\
\hline $9-1,141$ & 39.41 & 2.5 & 2.1 & (3) & 0.23 & 11 \\
\hline $10-2,144$ & 45.69 & 2.2 & 1.4 & 7 & & \\
\hline $10-3,103$ & 46.78 & 2.7 & 1.9 & 7 & & \\
\hline $11-1,121$ & 48.71 & 2.2 & 1.8 & (3) & 0.17 & 12 \\
\hline $481 \mathrm{~A}-1-1,136$ & 43.36 & 2.3 & 1.5 & 6 & & \\
\hline $2-1,70$ & 52.20 & 3.2 & 1.0 & 19 & & \\
\hline $3-1,109$ & 62.09 & 2.1 & 1.6 & (4) & 0.14 & 13 \\
\hline $4-2,74$ & 72.74 & 3.7 & 2.7 & (8) & 0.23 & 13 \\
\hline $4-2,105$ & 73.05 & 3.5 & 2.1 & 12 & & \\
\hline $5-6,26$ & 87.76 & 2.2 & 1.3 & 8 & & \\
\hline $6-3,134$ & 93.84 & 1.4 & 1.2 & (2) & 0.09 & 15 \\
\hline $7-4,125$ & 104.75 & 2.5 & 1.9 & 5 & & \\
\hline $8-2,114$ & 111.14 & 2.6 & 2.0 & 5 & & \\
\hline $8-6,29$ & 116.29 & 2.0 & 1.8 & (2) & 0.14 & 15 \\
\hline $9-5,30$ & 124.30 & 3.5 & 2.2 & 11 & & \\
\hline $10-1,53$ & 128.03 & 2.0 & 1.7 & 3 & & \\
\hline $10-2,104$ & 130.04 & 1.4 & 1.3 & (1) & 0.11 & 14 \\
\hline $11-3,144$ & 141.44 & 1.8 & 1.3 & 4 & & \\
\hline $12-1,107$ & 147.57 & 1.5 & 1.3 & (2) & 0.12 & 13 \\
\hline $12-4,53$ & 151.53 & 1.4 & 1.0 & 4 & & \\
\hline $13-1,132$ & 157.32 & 1.5 & 0.9 & (5) & 0.10 & 11 \\
\hline $13-5,25$ & 162.25 & 2.8 & 1.4 & 12 & & \\
\hline $14-3,50$ & 169.00 & 1.1 & 0.8 & (2) & 0.05 & 19 \\
\hline $14-4,2$ & 170.02 & 1.2 & 0.6 & 5 & & \\
\hline $14-4,52$ & 170.52 & 0.6 & 0.4 & (2) & 0.03 & 14 \\
\hline $18-1,27$ & 203.77 & 1.1 & 0.9 & (2) & 0.09 & 12 \\
\hline $20-1,60$ & 223.10 & 3.5 & 1.8 & 14 & & \\
\hline $20-2,22$ & 224.22 & 1.2 & 1.1 & (1) & 0.08 & 16 \\
\hline $22-1,100$ & 242.50 & 2.5 & 1.7 & 6 & & \\
\hline $22-5,132$ & 248.82 & 2.5 & 2.5 & (0) & 0.19 & 15 \\
\hline $23-1,11$ & 251.11 & 2.2 & 1.3 & 7 & & \\
\hline $24-3,126$ & 264.76 & 1.5 & 1.3 & (2) & 0.10 & 15 \\
\hline $25-6,10$ & 279.10 & 1.8 & 1.1 & 6 & & \\
\hline $26-4,64$ & 284.64 & 1.9 & 1.4 & (4) & 0.11 & 15 \\
\hline $27-2,38$ & 290.88 & 2.1 & 1.4 & 6 & & \\
\hline $27-3,61$ & 292.61 & 2.3 & 1.3 & 8 & & \\
\hline $28-3,131$ & 302.81 & 1.7 & 1.3 & (3) & 0.09 & 16 \\
\hline $29-1,133$ & 309.33 & 1.8 & 1.0 & 7 & & \\
\hline $30-2,137$ & 320.37 & 2.8 & 2.0 & (7) & 0.16 & 16 \\
\hline $30-4,129$ & 323.29 & 2.4 & 1.2 & 10 & & \\
\hline $30-5,46$ & 323.96 & 1.3 & 1.1 & (2) & 0.09 & 15 \\
\hline $30-6,5$ & 325.05 & 1.8 & 1.3 & 4 & & \\
\hline $30-6,46$ & 325.46 & 1.7 & 1.2 & (4) & 0.09 & 16 \\
\hline $31, \mathrm{CC}$ & 328.30 & 2.6 & 2.1 & (4) & 0.13 & 19 \\
\hline $33-1,20$ & 346.20 & 1.3 & 0.9 & (0) & 0.05 & 20 \\
\hline
\end{tabular}

a Total carbonates calculated as calcium carbonate; values in parentheses were determined by the carbonate bomb method. 